\title{
Three-dimensional computed topography analysis of a patient with an unusual anatomy of the maxillary second and third molars
}

\author{
Jin Zhao ${ }^{1}$, Yan $\mathrm{Li}^{2}$, Zhi-wei Yang ${ }^{3}$, Wei Wang ${ }^{4}$, Yan Meng ${ }^{1}$ \\ ${ }^{1}$ Department of Stomatology, First Affiliated Hospital of Xinjiang Medical University, Urumqi 830054, China; \\ ${ }^{2}$ Department of Stomatology, Fifth Affiliated Hospital of Xinjiang Medical University, Urumqi 830011, China; \\ ${ }^{3}$ Information Technology Division of Urumqi Railway Bureau, Urumqi 830011, China; ${ }^{4}$ Xinjiang KeLi Advanced \\ Manufacturing Technology Ltd., Urumqi 830000, China
}

We present a case of a patient with rare anatomy of a maxillary second molar with three mesiobuccal root canals and a maxillary third molar with four separate roots, identified using multi-slice computed topography (CT) and three-dimensional reconstruction techniques. The described case enriched/might enrich our knowledge about possible anatomical aberrations of maxillary molars. In addition, we demonstrate the role of multi-slice $\mathrm{CT}$ as an objective tool for confirmatory diagnosis and successful endodontic management.

Keywords: maxillary second molar; mesiobuccal root; maxillary third molar; multi-slice computed topography; three-dimensional reconstruction

International Journal of Oral Science (2011) 3: 225-228. doi: 10.4248/IJOS11078

\section{Introduction}

The complexity and diversity of root canal morphology, particularly in multi-rooted teeth, are constant challenges for diagnosis and successful endodontic therapy [1]. Thus, it is necessary for the clinician to have a knowledge of root canal anatomy and its variations [2]. The maxillary second molar commonly has three roots (two buccal and one palatal); the palatal and distobuccal roots have one canal and the mesiobuccal root has one or two canals [3-7]. Variations in root anatomy have been previously observed. Some have reported cases of maxillary second molar with two palatal roots [8-9]. Approximately $0.416 \%$ of maxillary second molars have four roots [10]. The researchers [11] have reported a maxillary second molar

*Correspondence: Jin Zhao

Tel: 869914326923 ; Fax: 869914365424

E-mail: merryljin@sina.com

Received 13 July 2010; Accepted 21 June 2011 with three buccal roots. Kottoor et al. [12] reported a maxillary second molar with five roots and five canals. To date, however, no one has reported the existence of three canals in the mesiobuccal root of the maxillary second molar in Xibo minority using multi-slice computed topography (CT) in endodontic therapy.

Owing to the nature of unpredictable configurations, few studies have described maxillary third molar aberrations [13]. The researchers [1] have found that the maxillary third molar presents various root forms, including three fused roots (37\%), two fused roots $(7 \%)$, four fused roots $(2 \%)$, and four separated roots (less than $2 \%$ ). Conventional radiographs are routinely used to assess root canal anatomy, but because of their inherent limitations, conventional radiographs cannot definitively locate the root canal, and hence, cannot be used in the management of complicated cases. As a powerful diagnostic tool, spiral computed topography (SCT) has overcome the disadvantages of conventional radiography, and has attracted a great amount of attention in endo- 
dontic therapy [14-16].

The purpose of this report is to present three-dimensional (3D) images of two maxillary molars with rare anatomical findings, and to demonstrate the role of multi-slice CT as a confirmatory diagnostic tool in endodontic management.

\section{Case Report}

A 40-year-old Xibo minority female patient, who had partial dentures and moderate periodontitis, was referred to our hospital for a conus crown telescopic denture.

During root canal treatment of the abutment tooth, we identified a fissure on the mesiobuccal side of the pulp chamber floor of the right maxillary second molar between the mesiobuccal and palatal canal orifices. After thoroughly probing the fissure, we identified a second mesiobuccal canal closely approximated to the mesiobuccal orifice and a third canal orifice, leading to another root canal of the the mesiobuccal root between MB2 and the palatal canal orifice. An off-angle radiograph was acquired with size $15 \mathrm{~K}$-files in place. The radiograph clearly revealed three separate canals (mesiobuccal, the second mesiobuccal, and the third mesiobuccal) in the mesiobuccal root (Figure 1A). After the working length was determined with the help of the apex locator, Root
ZX (Morita, Tokyo, Japan) and a radiograph (Figure 1B), coronal flaring for all five canals was performed with Gates Glidden drills. The canals were cleaned and shaped to F1 (mesiobuccal and distal buccal) and F2 (palatal) by the crown-down technique using the Protaper rotary instrument (Dentsply Maillefer, Ballaigues, Switzerland) and obturated by cold lateral compaction of the guttapercha and AH Plus sealer (Dentsply Maillefer, Ballaigues, Switzerland). A postoperative radiograph was obtained (Figure 1C), but it only provided a two-dimensional image of the root canal, thereby resulting in superimposed images. To confirm the obturation and ascertain the rare root canal anatomy of the maxillary second molar, and the status of other abutment teeth in a 3D manner, dental imaging with the aid of a multi-slice CT was planned. Informed consent was obtained from the patient. Axial cross-section images of the maxilla were obtained using a LightSpeed multi-slice CT (GE Company, USA). The scanning parameters were as follows: current, $250 \mathrm{~mA}$; voltage, $120 \mathrm{kV}$; helical scanning, $1.0 \mathrm{~s} \cdot \operatorname{rot}^{-1}$; and slice thickness, $0.625 \mathrm{~mm}$. The computed topographic slice images were reconstructed using a high-resolution bone algorithm with the GE post-processing software and were used as the basic data for $3 \mathrm{D}$ reconstruction (Figure 2). The areas representing the tooth were segmented out from each slice by using the Mimics 10.0
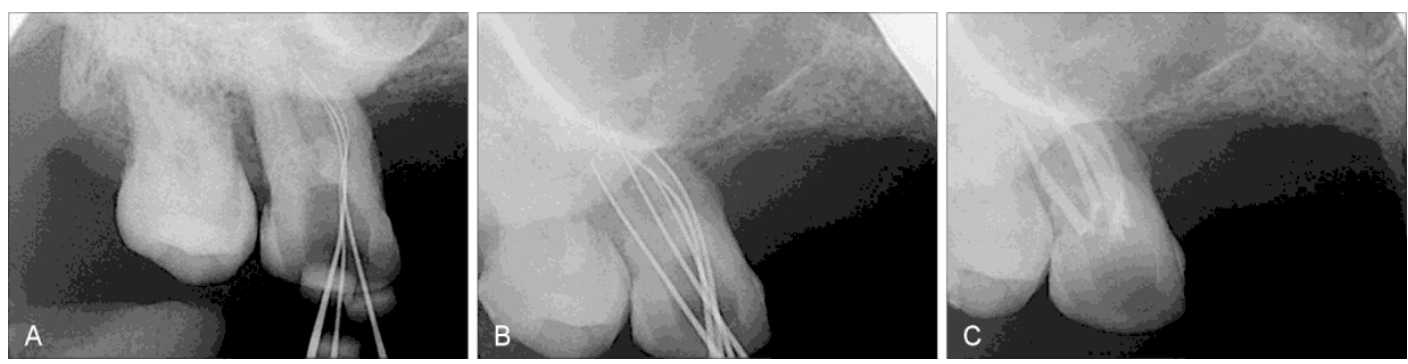

Figure 1 (A) Preoperative radiograph. Location of the three root canals in the mesiobuccal root with files. (B) Working length determination. (C) Postobturation radiograph.
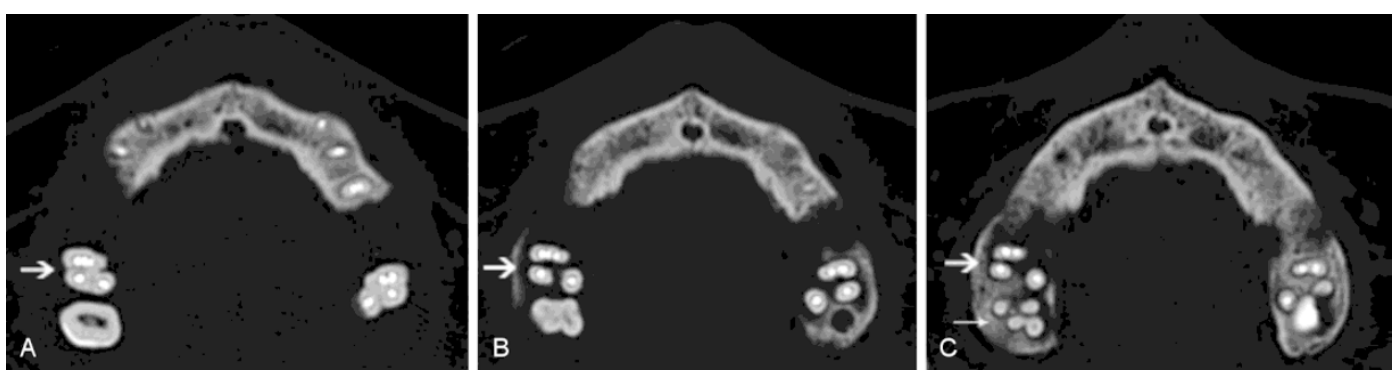

Figure 2 Cross-section reconstruction of the maxillary molar. (A) Coronal third section of the roots of the obturated maxillary second molar (arrow). (B) Middle third section of the roots of the obturated maxillary second molar (arrow). (C) Apical third section of the roots of the obturated maxillary second molar and third molar (arrows). 
software (Macrovision Corporation, Santa Clara, USA). The primary reconstructed models in STL format were uploaded to Geomagic studio 8.0 (PTC, Needham, USA) for further refinement and thinning. The final $3 \mathrm{D}$ visual models of the teeth and root canals were obtained using the NX software (Siemens, Tarrytown, USA). The models revealed that the mesiobuccal root of the right maxillary second molar had three root canals, and the mesiobuccal and mesiocentral canals were fused at the apical third (Figure 3). The third molar had four separated roots (Figure 4).
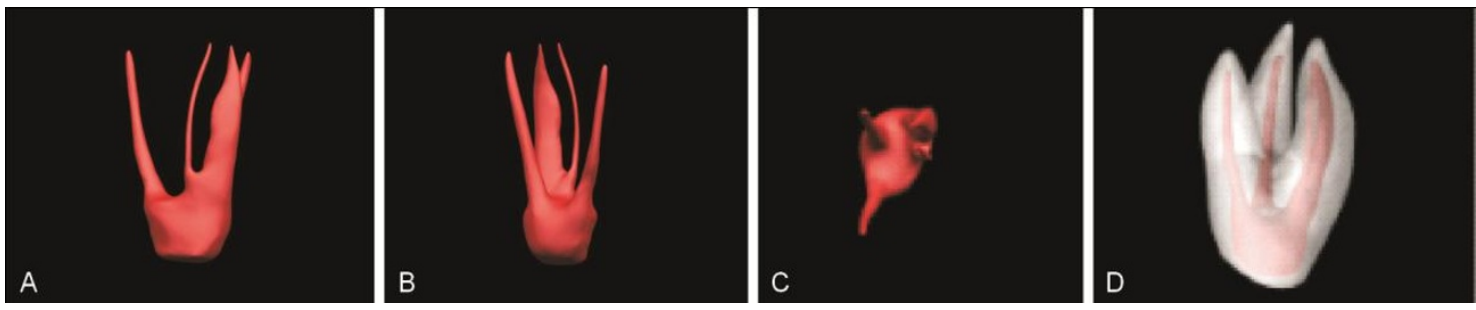

Figure 3 3D reconstruction of the obturated maxillary second molar of the root canal system. (A) Mesial view of the root canal system. (B) Distal view of the root canal system. (C) Apical view of the root canal system. (D) 3D transparent model of the obturated maxillary second molar.
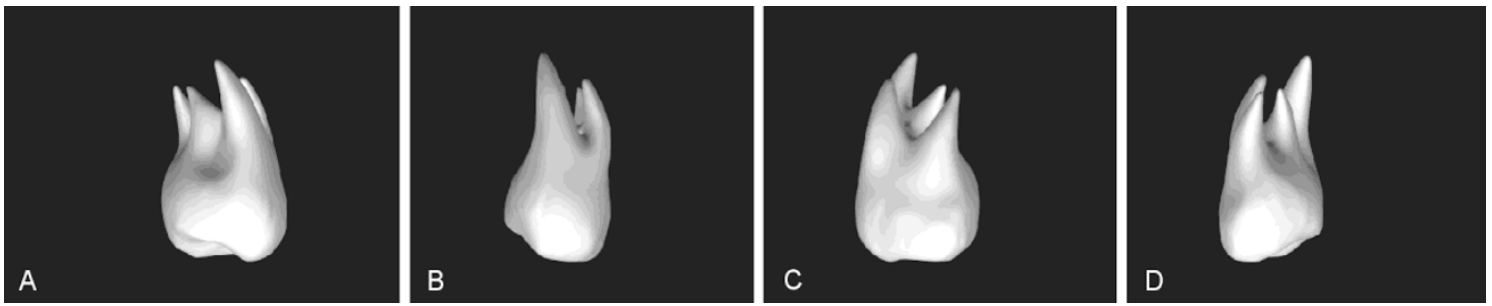

Figure 4 3D reconstruction of the maxillary third molar with four separated roots. (A) Mesial view. (B) Palatal view.

(C) Distal view. (D) Buccal view.

\section{Discussion}

According to Vertucci's classification [17], there are eight types of root canal configurations in human permanent teeth, among which type VIII and its additional modifications are the rarest and most complex. There are only a few case reports on maxillary dentition [17-22]. Three root canals are commonly found in the mesiobuccal root of the maxillary first molar and the premolar, but their proper diagnoses and nonsurgical endodontic therapy are rarely successful on the first attempt. For accurate diagnosis, the clinician must have a thorough understanding of the anatomical variations revealed by various detection methods. Additional off-angle radiographs and specific magnifying devices are important diagnostic tools for locating additional canals [2, 20-21, 23]. Interpretation and appraisal based on a two-dimensional radiograph may alert the clinician to the presence of aberrant anatomy; however, these techniques do not provide $3 \mathrm{D}$ images of the teeth and are not able to present the variable morphological structure of the root canals and their interrelations. CT scanning techniques reveal the variations in root anatomy through multiplanar reconstruction, and assist the clinician in making a confirmatory diagnosis. CT provides accurate positioning, that is non-invasive, reproducible, and of high resolution. The reconstructed images based on CT image reliably represent the original structure.

The researchers [14-16, 23-24] have reported cases of successful endodontic diagnosis and therapy using SCT. On the basis of previous reports, multi-slice CT was planned for a final diagnosis of the anatomic variations of the maxillary second molar. The 3D images of the three mesiobuccal root canals of the right maxillary second molar were reconstructed using multi-slice CT and 3D reconstruction techniques. Mimics 10.01 is considered as an effective interactive digital tool for revealing, segmenting, and reconstructing CT images. With the help of Mimics, the varying morphology of root cananls can be reconstructed and presented from different views. 
Furthermore, reconstructed CT images in this study have also revealed have another anatomic variation: four separate roots were identified in the right third molar. With an increased demand for prosthetic dentistry, retention of the third molar may become critical for the success of the overall treatment plan. However, note that the resolution of multi-slice $\mathrm{CT}$ is limited, and cannot provided the fine structures of the periapical section insufficiently. With rapid advancements in sensor technology, cone-beam computed tomography (CBCT), a new technique based on volumetric tomography, has increased accuracy and sub-millimeter resolution, and achieved dose reduction.

\section{Conclusion}

In the present case, we visualized the unusual anatomy of a maxillary second molar with three root canals in the mesiobuccal root and a third molar with four separated roots after $3 \mathrm{D}$ digital reconstruction. The multi-slice CT images obtained from multiple angles helped the clinician understand the complex root canal configurations of the abnormal teeth and increased our knowledge regarding possible anatomical aberrations of the maxillary molars. Findings from this study indicate that multi-slice CT is a valuable tool that provides accurate $3 \mathrm{D}$ imaging information for the clinician in endodontic treatment.

\section{References}

1 Malagnino V, Gallotini L, Passsariello P. Some unusual clinical cases on root canal anatomy of permanent maxillary molars. J Endod 1997; 23: 127-128.

2 Baratto-Filho F, Fariniuk LF, Ferreira EL, et al. Clinical and macroscopic study of maxillary molars with two palatal roots. Int Endod J 2002; 35: 796-801.

3 Kulild JC, Peters DD. Incidence and configuration of canal systems in the mesiobuccal root of maxillary first and second molars. J Endod 1990; 16: 311- 317.

4 Gilles J, Reader A. An SEM investigation of the mesiolingual canal in human maxillary first and second molars. Oral Surg Oral Med Oral Pathol 1990; 70: 638-643.

5 Eskoz N, Weine FS. Canal configuration of the mesiobuccal root of the maxillary second molar. $J$ Endod 1995; 21: 38-42.

6 Stropko JJ. Canal morphology of maxillary molars: clinical observations of canal configurations. J Endod 1999; 25: 446-450.

7 Alavi AM, Opasanon A, Ng YL, Gulabivala K. Root and canal morphology of Thai maxillary molars. Int Endod $J$ 2002; 35: 478-485.
8 Barbizam JV, Ribeiro RG, Filho MK. Unusual anatomy of permanent maxillary molars. $J$ Endod 2004; 30: 668-671.

9 Deveaux E. Maxillary second molar with two palatal roots. J Endod 1999; 25: 571-573.

10 Libfeld H, Rotstein I. Incidence of four-rooted maxillary second molars: literature review and radiographic survey of 1200 teeth. J Endod 1989; 15: 129-131.

11 Fahid A, Taintor JF. Maxillary second molar with three buccal roots. J Endod 1988; 14: 181-183.

12 Kottoor J, Hemamalathi S, Sudha R, Velmurugan N. Maxillary second molar with 5 roots and 5 canals evaluated using cone beam computerized tomography: a case report. Oral Surg Oral Med Oral Pathol 2010; 109: 162-165.

13 Sidow SJ, West LA, Liewehr FR, Loushine RJ. Root canal morphology of human maxillary and mandibular third molars. J Endod 2000; 26: 675-678.

14 Sponchiado EC Jr, Ismail HA, Braga MR, de Carvalho FK, Simoes CA. Maxillary central incisor with two root canals: a case report. J Endod 2002; 93: 328-332.

15 Gopikrishna V, Bhargavi N, Kandaswamy D. Endodontic management of a maxillary first molar with a single root and a single canal diagnosed with the aid of spiral CT: a case report. J Endod 2006; 32: 687-691.

16 Ballal S, Sachdeva GS, Kandaswamy D. Endodontic management of a fused mandibular second molar and paramolar with the aid of spiral computed tomography: a case report. J Endod 2007; 33: 1247-1251.

17 Vertucci FJ. Root canal anatomy of the human permanent teeth. Oral Surg Oral Med Oral Pathol 1984; 58: 589-599.

18 Martinez-Berna A, Ruiz-Badanelli P. Maxillary first molars with six canals. $J$ Endod 1983; 9: 375-380.

19 Walvekar SV, Behbehani JM. Three root canals and dens formation in a maxillary lateral incisor: a case report. $J$ Endod 1997; 23: 185-186.

20 Maggiore F, Jou YT, Kim S. A six-canal maxillary first molar: case report. Int Endod J 2002; 35: 486-491.

21 Soares JA, Leonardo RT. Root canal treatment of threerooted maxillary first and second premolars - a case report. Int Endod J 2003; 36: 705-710.

22 Ferguson DB, Kjar KS, Hartwell GR. Three canals in the mesiobuccal root of a maxillary first molar: a case report. $J$ Endod 2005; 31: 400-402.

23 Lee SJ, Jang KH, Spangberg LW. Three-dimensional visualization of a mandibular first molar with three distal roots using computer-aided rapid prototyping. Oral Surg Oral Med Oral Pathol 2006; 101: 778-774.

24 Sachdeva GS, Ballal S, Gopikrishna V, Kandaswamy D. Endodontic management of a mandibular second premolar with four roots and four root canals with the aid of spiral computed tomography: a case report. J Endod 2008; 34: 104-107. 